\title{
Synthesis and Characterization of Low Dielectric Polymer Composite: Ethylene- Propylene-Diene Terpolymer/ Barium hexa ferrite
}

\author{
Deeptimayee Khatua, R. N. P.Choudhary, P.Ganga Raju Achary
}

\begin{abstract}
A series of organic- inorganic polymer composites were synthesized by incorporating Barium hexa ferrite $(\mathrm{BHF})$ at different $w t \%$ in the Ethylene-propylene-diene terpolymer (EPDM) polymer matrix by adopting solution casting method. The synthesized $\mathrm{EPDM} / \mathrm{BHF}$ polymer composite were characterized through different techniques i.e.; X-ray diffraction study, SEM(scanning electron microscope), dielectrical and electrical behavior in the frequency range $1 \mathrm{kHz}$ to $1 \mathrm{MHz}$. A detailed study of the dielectric behavior i.e.; $\varepsilon$ ' (dielectric permittivity), tan $\delta$ (dielectric loss), M'and $M$ ', (dielectric modulus) and aac (electrical conductivity) were reported in this communication. The XRD of the composites showed the uniform distribution of the filler particles in the polymer matrix. The SEM micrograph confirms the homogeneous dispersion and distribution of BHF particles in the EPDM polymer matrix. These polymer composite show a promising dielectric behavior which is different from its constituent components.
\end{abstract}

Keywords: Ethylene-propylene-diene, barium hexaferrite, Dielectric properties.

\section{INTRODUCTION}

Recent years the development of polymeric nano composite has made an important contribution to electrical industry. Polymer offers various advantages like light weight, high mechanical strength to weight ratio, high resistance, high voltage resistance and ease of fabrication and processing than the traditional inorganic materials [1].

EPDM is a high density synthetic rubber containing two monomers such as ethylene and propylene .The nonpolar structure of EPDM provides excellent electrical properties and its backbone helps the polymer resistant to oxidation and thermal aging and easy to process at low temperature. This rubber is widely used for various outdoor application like automotive weather-stripping and seals, radiators, electrical insulations, roofing membranes, tubing, production of conveying belts etc. due to their versatile characteristics like heat and stress resistance, hardness, flexibility, chemical resistance to polar media, good electrical characteristics. 2].It possess the lowest specific gravity among all rubbers, hence it can replace all other elastomers like styrene-butadiene, nitrile, Buna- $\mathrm{N}$ etc.[3] .Synthetically produced EPDM is expensive and it cannot be used as articles directly their softness. Hence they have to

Revised Manuscript Received on September 14, 2019.

Deeptimayee Khatua, Department of Chemistry, Siksha 'O' Anusandhan, Deemed to be University, Khandagiri, Square, Bhubaneswar751030, Odisha, India

R. N. P.Choudhary, Department of Physics, Siksha O Anusandhan, Deemed to be University, Khandagiri, Square, Bhubaneswar, Odisha, India.

P.Ganga Raju Achary, Department of Chemistry, Siksha 'O' Anusandhan, Deemed to be University, Khandagiri, Square, Bhubaneswar751030, Odisha, India. (Email: pgrachary@soa.ac.in) modify with inorganic filler to enhance the mechanical, electrical and dispersion properties [4]. The filler may be conductive or nonconductive ceramics, nanoparticles, ferroelectric materials etc. In this present work Barium hexaferriet $(\mathrm{BaFe} 12 \mathrm{O} 19)$ is taken as filler which has high value magnetization and intrinsic coerctivity. It can widely use as permanent magnet, in high density recording application and magneto optical devices. [5]

Research on EPDM composites have been carried out by many authors. Ali et al. investigated that the thermal and mechanical behavior of EPDM matrix increases with silica loading and it possess insulating properties which is helps them in high voltage insulation application.[2] The dielectric relaxation behavior of $\mathrm{EPDM} / \mathrm{xGnP}$ nano composite was studied by Dash et al. It was reported that the dielectric permittivity and ac conductivity of the sample increases with filler loading. [6] Su et al. was studied the effect of chemical modification with different coupling agent on EPDM/barium titanate polymer composite. The surface modification of $\mathrm{BaTiO} 3$ with coupling agents introduce the functional groups on the particle and hence the polar surface tension of the particle decreases. And er (dielectric constant) and $\tan \delta$ (tangent loss) of EPDM composite decreases with increase in modified filler loading [7].Kurata et al. reported that by addition of Alumina trihydrates (ATH) as filler, the electrical tracking resistance of EPDM increases [8].Ismail et al. was studied the effect of the white fillers (Kaoline, quartz, PVC and talc) and two vulcanizing systems (TMTD and S/CBS) on physicomechanical properties of EPDM matrix before and after aging [9].

The electrical behavior of the polymer of the composite depends upon the polymer matrix, filler added, dispersion of filler in the polymer, frequency and temperature [5]. This work was devoted to studying the dielectric and electric behavior of EPDM / BHF composites at wide frequency range $(1 \mathrm{kHz}$ to $1 \mathrm{MHz})$ at different temperature.

\section{EXPERIMENT}

\section{Synthesis of BaFe12O19-EPDM Nano composite films}

EPDM (Ethylene propylene diene terpolymer) and Cyclohexane (\%) were purchased from LOBA chemicals. The filler barium hexa ferrite (BHF) Nano particles was previously prepared by using Sol gel combustion method via urea root [5].EPDM/BHF Nano composites were prepared 
by adopting solution casting method. First the EPDM rubber was completely dissolved with cyclohexane through magnetic stirrer. Then different amount of BHF was dispersed in the EPDM solution with the help of ultrasonication. After the complete dispersion of the filler, it was poured in a Petridis for casting. The sample on the Petridis was kept undisturbed and dried to evaporate the solvent. After complete evaporation of the solvent the film was peeled out from the Petridis. By applying the above method polymer composite of different wt $\%(0,2.5,5,7.5$ and 10 wt. $\%)$ were prepared.

\section{RESULTS AND DISCUSSION}

\subsection{X-ray diffraction analysis}

Previously we prepared the ferrite sample and its phase quantification was done by rietveld refinement method using MAUD software. The BHF sample contained $90.91 \%$ of BaFe12019

and $7.086 \%$ of F2O3 as impurity with sig value 1.9. The sample exhibited hexagonal structure with lattice parameter $\mathrm{a}=\mathrm{b}=5.8950334 \AA$ and $\mathrm{c}=23.21821 \AA$ [5].

Fig 1(c) indicates the XRD diffraction of the polymer composite films at different wt\% of the BHF. Due to the amorphous nature of the EPDM polymer a broad peak is obtained at $2 \theta=20.93^{\circ}$. In case of higher filler content the broadness of the amorphous peak of EPDM gradually decreases. The XRD reflection appears at higher concentration (i.e; $5 \mathrm{wt} \%, 7.5 \mathrm{wt} \%, 10 \mathrm{wt} \%$ ) of the EPDM polymer composite which concludes that with the introduction of filler in the polymer matrix increases the crystalline structure of the polymer matrix.

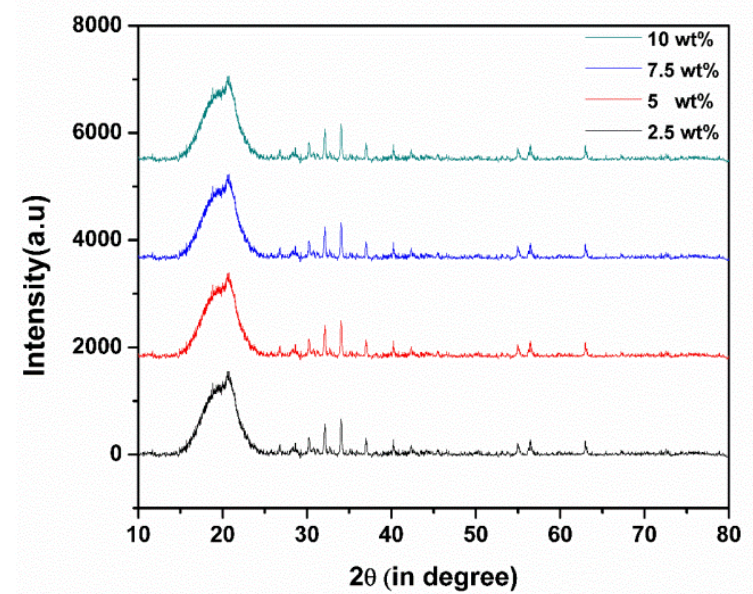

Fig. 1 (a) XRD reflection of polymer composite at different wt\%

\subsection{SEM Analysis}

BHF dispersion in the EPDM polymer matrix at different wt\% was confirmed by SEM study which was shown in Fig.2 (a)(b)(c)(d) and(e) respectively.SEM micrographs of the all the composites show Schallamach type wavy appearance[10]. As seen, homogeneous dispersion and distribution of BHF particles are shown through the EPDM matrix. It also indicates the proper de-agglomeration of nanoparticles in polymer. But at higher wt\% (10 wt \%) Fig.2 (e) the BHF particles are agglomerate hence pores are created in the composite.
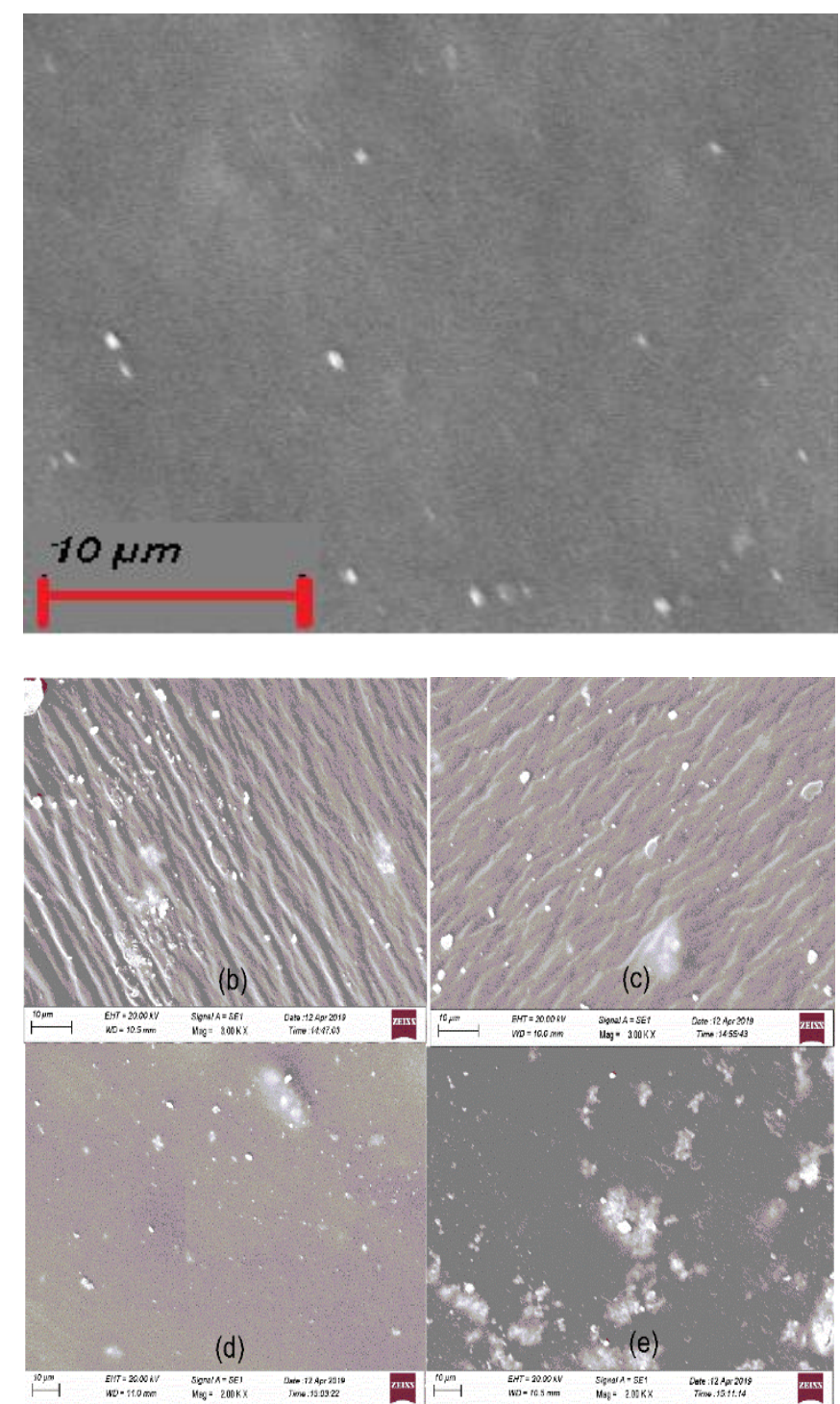

Fig 2. SEM micrographs of EPDM/BHF composite(a) 0 wt\%; (b) 2.5 wt\%; (c) 5 wt\%, (d)7.5 wt\% and (e) 10 wt\%

\subsection{Dielectric properties}

Dielectric response of a polymeric composite material can be understood by detailed analysis of er (dielectric constant) and $\tan \delta$ (dielectric loss). The dielectric constant of the EPDM polymer composite was determined by using the following equation,

$$
\varepsilon r=\frac{\mathrm{Cl}}{\mathrm{A} \varepsilon 0},[11]
$$

where $\varepsilon r$ is the dielectric constant of the polymer, $\varepsilon 0$ is the dielectric permittivity in vacuum i.e; $8.854 \times 10-12 \mathrm{~F} \mathrm{~m}-1$, $\mathrm{C}$ is the capacitance of composite, $\mathrm{A}$ is the overlapping area of two silver electrode and 1 is the thickness of the polymer sample. Fig 3 shows the variation of dielectric permittivity of the composites with frequency at room temperature. Generally the value of dielectric permittivity decreases with increase in frequency. But all the composites have constant dielectric permittivity with increase in frequency. Whereas, 
the permittivity of the samples gradually decreases with filler loading up to $5 \mathrm{wt} \%$ then the value increases with the higher filler loading (Fig 5(a)). The permittivity of $0 \mathrm{wt} \%, 2.5$ wt $\%, 5 \mathrm{wt} \%, 7.5 \mathrm{wt} \%$ and $10 \mathrm{wt} \%$ at $1 \mathrm{kHz}$ are $2.20,1.98,1.65,1.686$ and 1.790 respectively. The decreasing tendency of dielectric permittivity can be explained by using Debye equation

$$
\frac{k-1}{k+2}=\frac{4 \pi}{3} N(\alpha e+\alpha d+\mu 2 / 3 \mathrm{kBT})[12]
$$

Where $\mathrm{k}$ is the dielectric permittivity of the material, $\mathrm{N}$ is the number of dipoles, $\alpha \mathrm{e}$ is the electronic polarization $\alpha \mathrm{d}$ is the distortion polarization $\mathrm{kB}$ is the Boltzmann constant.

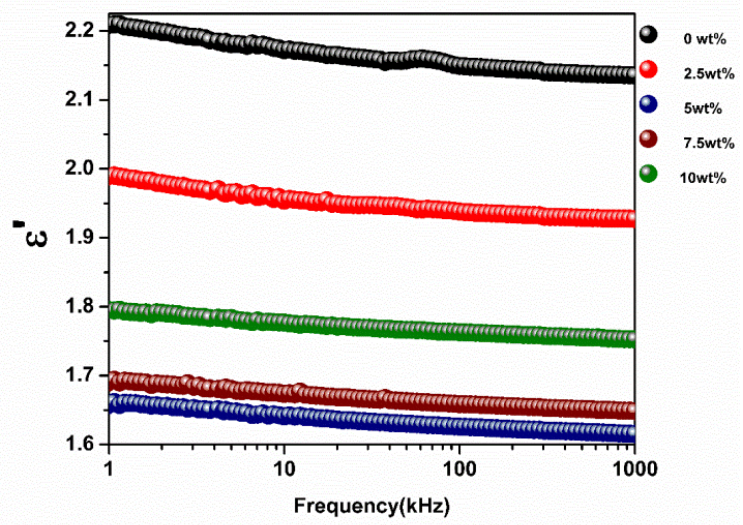

Fig 3. Frequency vs. dielectric permittivity ( $\left.\varepsilon^{\prime}\right)$ at $25^{\circ} \mathrm{C}$

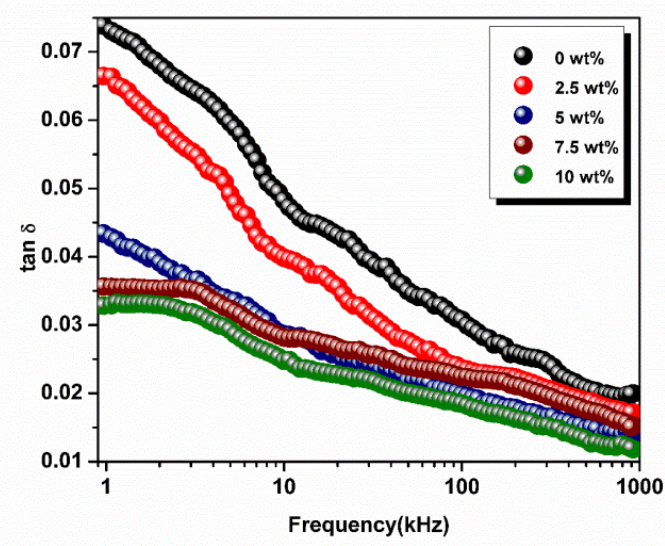

Fig 4. Frequency vs. dielectric loss $(\tan \delta)$ at room temperature

Fig 4. depicts the effect of frequency on tangent loss factor $(\tan \delta)$ of the EPDM polymer matrix and its composite with BHF.The value of the dielectric loss factor gradually decreases with increase in frequency hence the sample can be used as an ideal capacitor. Fig 5(b) shows the effect of filler loading in the EPDM polymer matrix at different frequency range i.e; $1 \mathrm{kHz}, 10 \mathrm{kHz}$, and $1 \mathrm{MHz}$. The dielectric loss or tangent loss of a composite is affected by crystallinity, density, uniform distribution of filler in the matrix and interaction between the filler and the polymer matrix.[13] From the XRD diffraction pattern it can be observed that the crystallinity increases with increase in BHF filer loading .hence the dielectric loss of the sample decreases. This trend continuous up to $0 \mathrm{wt} \%$ to $5.5 \mathrm{wt} \%$ (Fig 3(b)) afterwards it increases slightly with higher filler loading. It is observed from Fig 4 that at higher frequency region the dielectric properties are independent on frequency but at low frequency region the dielectric permittivity and tangent loss show non Debye behavior i.e.; it decreases with rise in frequency. This behavior of the dielectric property is described by Koop's model. According to this model at low frequency region the dielectric constant is due to the grain boundaries which possess high dielectric constant. Whereas at higher frequency the dielectric constant (cr) is due to grain which have low value.[14]

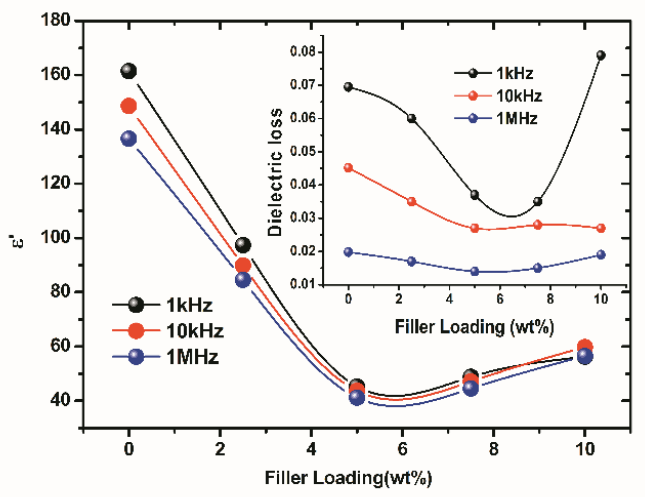

Fig. 5(a) Filler loading vs. dielectric permittivity (b) Filler loading vs. dielectric loss plot at different frequency range.

The value of dielectric permittivity and dielectric loss at room temperature and different frequency range is reported in Table 1.

Table 1: Dielectric properties of the sample at different frequency

\begin{tabular}{|c|c|c|c|c|c|c|}
\hline \multirow{2}{*}{$\begin{array}{l}\text { Freque } \\
\text { ncy } \\
\text { BHF } \\
\text { wt\% }\end{array}$} & \multicolumn{2}{|l|}{$1 \mathrm{kHz}$} & \multicolumn{2}{|l|}{$10 \mathrm{kHz}$} & \multicolumn{2}{|l|}{$1 \mathrm{MHz}$} \\
\hline & $\begin{array}{l}\varepsilon ' \\
\text { (Dielectri } \\
\text { c } \\
\text { Permittiv } \\
\text { ity) }\end{array}$ & $\begin{array}{l}\tan \delta \\
\text { (Tange } \\
\text { nt loss) }\end{array}$ & $\begin{array}{l}\varepsilon ' \\
\text { (Dielectri } \\
\text { c } \\
\text { Permittiv } \\
\text { ity) }\end{array}$ & $\begin{array}{l}\tan \delta \\
\text { (Tange } \\
\text { nt loss) }\end{array}$ & $\begin{array}{l}\varepsilon^{\prime} \\
\text { (Dielectri } \\
\text { c } \\
\text { Permittiv } \\
\text { ity) }\end{array}$ & $\begin{array}{l}\tan \delta \\
\text { (Tange } \\
\text { nt loss) }\end{array}$ \\
\hline 0 & 2.20 & 0.0738 & 2.172 & 0.0482 & 2.135 & 0.0198 \\
\hline 2.5 & 1.988 & 0.6647 & 1.9539 & 0.0398 & 1.927 & 0.0171 \\
\hline 5 & 1.656 & 0.0431 & 1.640 & 0.0286 & 1.615 & 0.014 \\
\hline 7.5 & 1.689 & $\begin{array}{l}0.0356 \\
4 \\
\end{array}$ & 1.67368 & 0.0281 & 1.648 & 0.0150 \\
\hline 10 & 1.796 & 0.0328 & 1.7769 & 0.0247 & 1.752 & 0.011 \\
\hline
\end{tabular}

\subsection{Electrical conductivity}

Fig 6.depicts the variation of $\sigma a c$ (ac conductivity) with frequency at $25^{\circ} \mathrm{C}$. The ac conductivity of the composite can be evaluated by using the following equation $\sigma a c=\omega \varepsilon 0 \varepsilon r$ $\tan \delta$, where $\omega(=2 \pi \mathrm{f}), \varepsilon 0$ is the dielectric permitivity of vacuum [15]. The magnitude of $\sigma a c$ increases with rise in logarithmic frequency. Because at higher frequency the conductivity is due to ac contribution, which increases with rise in frequency. At lower frequency range the ac conductivity dominated by dc conductivity which is independent of frequency. The ac conductivity decreases

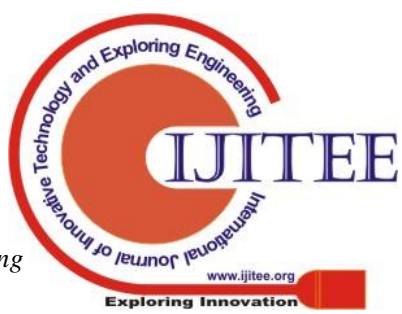


and turns more insulating with increase in filler loading up to $5 \mathrm{wt} \%$ then the value increases slightly with higher concentration of filler loading. The ac conductivity of the polymer composite can be explained by hoping mechanism. The value of ac conductivity increases with filler loading up to 5 wt $\%$ then it decreases.

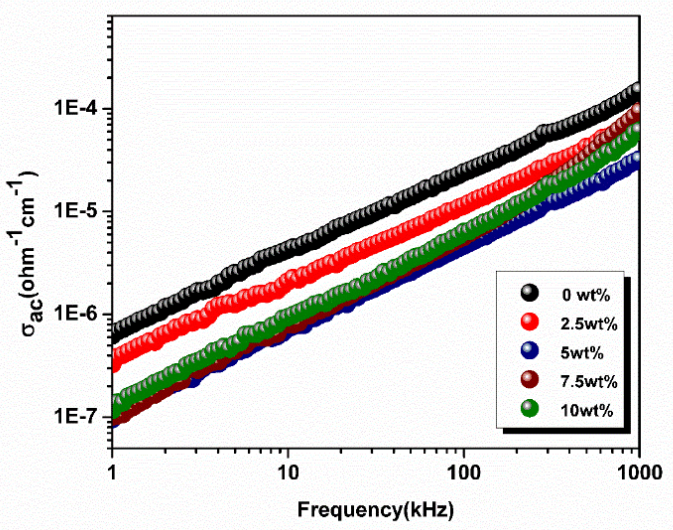

Fig 6 Frequency vs. ac conductivity at room temperature

\subsection{Electrical modulus}

From the of electrical modulus study we can measure the dielectric relaxation of a polymer composite. It is the defined as the complementary of dielectric permittivity $\mathrm{M}=$ $1 /$ er which can also be written as, $M *(\omega)=M^{\prime}+\mathrm{iM}^{\prime \prime}$, Where $\mathrm{M}^{\prime}$ and $\mathrm{M}^{\prime}$ 'is real and imaginary modulus of the system respectively. $\mathrm{M}^{\prime}=\varepsilon^{\prime} /\left(\varepsilon^{\prime} 2+\varepsilon{ }^{\prime} 2\right), \mathrm{M}^{\prime \prime}=\varepsilon^{\prime \prime} /\left(\varepsilon^{\prime} 2+\varepsilon^{\prime}{ }^{\prime} 2\right)$. [16] The variation of real electrical modulus with wide range of frequency is shown in the fig. 7(a).The real modulus of all composites increases linearly with rise in frequency and filler loading up to $5 \mathrm{wt} \%$. Then at higher filler loading the value of real modulus gradually deceases. Fig.7 (b) shows the effect of frequency change on imaginary part of electrical modulus. The magnitude of imaginary modulus decreases with rise in frequency. Whereas the value increases with filler loading up to $5 \mathrm{wt} \%$, then the value decreases at higher filler loading.

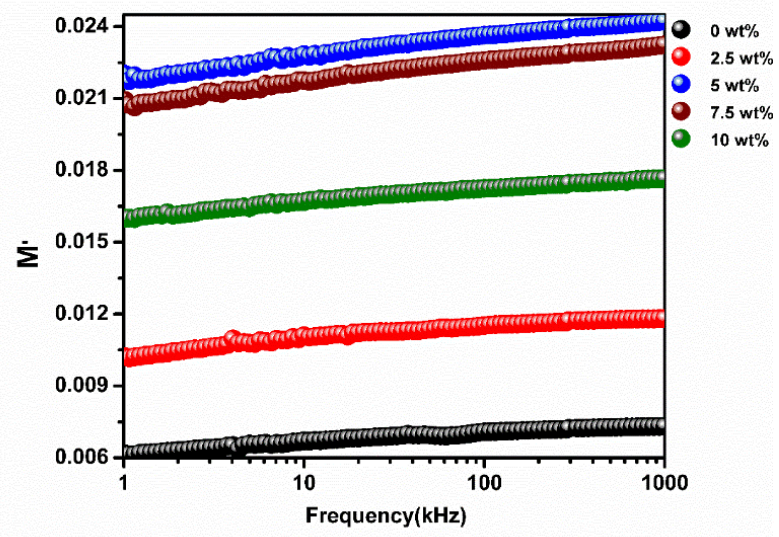

Fig 7(a) Frequency vs. Real modulus at room temperature

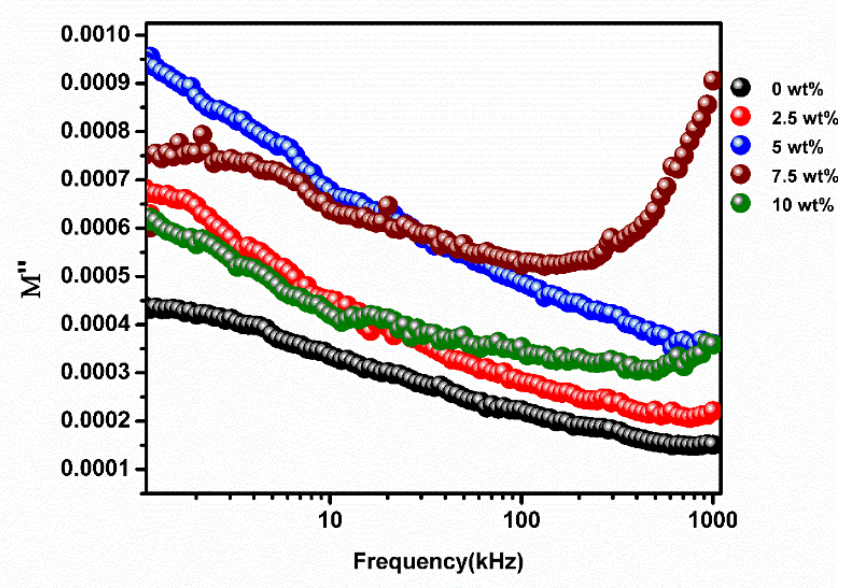

Fig 7(b) Frequency vs. Imaginary part of modulus at room temperature

\section{CONCLUSION}

EPDM/Barium hexaferriet nanocomposites at different wt $\%$ (0 wt $\%, 2.5 \mathrm{wt} \%, 5 \mathrm{wt} \%, 7.5 \mathrm{wt} \%$ and $10 \mathrm{wt} \%)$ were synthesized successfully by solution casting method and their structural morphological and dielectric properties are evaluated. The XRD reflection appears at higher concentration concludes that with the introduction of filler increases the crystalline structure of the polymer matrix. The SEM micrograph confirms the homogeneous dispersion and distribution of BHF particles in the EPDM polymer matrix. The value dielectric permittivity decreases with filler loading up to $5 \mathrm{wt} \%$. This phenomenon can be describe by Debye equation. In summary EPDM/Barium hexaferrite nano composite is low permittivity, low loss and flexible material which have potential for application in many electronic industry.

\section{ACKNOWLEDGEMENTS}

The present work is funded by the UGC-DAE-CSR, Mumbai (CRS-M-297).

\section{REFERENCES}

1. Vijayalekshmi A \&Majeed S, IJERA, 3(2013)1177-1180

2. Ali M \& Choudhry M, Materials Science-Poland, 33 (2015)213-219

3. Bhuvaneswari C,Kakade S ,Deuskar V ,Dange A \&Gupta M, Defence Science Journal, 58(2008) 94-102

4. $\mathrm{Hu} \mathrm{X}$, Gao H ,Zhou X ,Cui Y \& Ge H, RSC Advances,4(2014)13662-13668

5. Khatua D, Choudhary R N P \& Achary P, J. Mater. Sci.: Mater. Electron,29(2018)19425-19434

6. Dash B ,Achary P, Nayak N \&Choudhary R N P, J. Electron. Mater,46(2017)563-572

7. Su J \& Zhang J, RSC Advances,5(2015)78448-78456

8. Kurata Y ,Takano K ,Sakuraba K \& Hayashi M,IEEE,(2002)471-474

9. Ismail M \& Turky G, Polymer-Plastics Technology and Engineering,40(2001)635-652 
10. Mukhopadhyay A, Polymer testing,52(2016)167-173

11. Bei R, Qian C ,Zhang Y, Chi Z, Liu S ,Chen X Xu J \& Aldred M, J. Mater. Chem. C,5(2017)12807-12815

12. Sun H, Lv Y,Zhang C, Zuo X ,Li M ,Yue X \& Jiang Z, RSC Advances ,6(2018)7753-7760

13. Bharath R, Chakraborthy $\mathrm{T}$, Nhalil $\mathrm{H}$,Masin $\mathrm{B}$,Ashok $\mathrm{K}$ ,Sreemoolanadhan $\mathrm{H}$, Oommen C \& Elizabeth S, J. Mater. Chem. C,7(2019)4484-4496

14. Maji P, Pande P \& Choudhary R N P, Bull. Mater. Sci, 38(2015) 417-424

15. Dehury S, Achary P \& Choudhary R N P, J. Mater. Sci.: Mater. Electron,29(2018)3682-3689

16. Achary P, Dehury S \& Choudhary R N P, J. Mater. Sci.: Mater. Electron,29(2018)6805-6816

\section{FIGUER LEGENDS}

Fig. 1 (a) XRD reflection of polymer composite at different wt $\%$

Fig 2. SEM micrographs of EPDM/BHF composite(a) 0 wt $\%$; (b) $2.5 \mathrm{wt} \%$; (c) $5.0 \mathrm{wt} \%$,(d) $7.5 \mathrm{wt} \%$ and (e) $10 \mathrm{wt} \%$

Fig 3 Frequency vs. dielectric permittivity at room temperature

Fig 4 Frequency vs. dielectric loss at room temperature

Fig. 5(a) Filler loading vs. dielectric permittivity (b) Filler loading vs. dielectric loss plot at different frequency range.

Fig 6 Frequency vs. ac conductivity at room temperature

Fig 7(a) Frequency vs. Real modulus at room temperature

Fig 7(b) Frequency vs. Imaginary part of modulus at room temperature

\section{TABLE}

Dielectric properties of the sample at different frequency 